\title{
Investigating the Cardiac Effects of New Generation Anti-Diabetic Drug Empagliflozin Using Zebrafish Embryo Model
}

Fatima. K Alkawari', Wigdan. A Ali ${ }^{1}$

Supervised by: Dr. Huseyin C. Yalcin ${ }^{2}$, Dr. Fatiha Benslimane ${ }^{2}$

1Biomedical Science Department, College of Health Sciences, Qatar University, Doha, Qatar

\section{Abstract}

Type 2 diabetes mellitus (T2DM) affects $>16 \%$ of adults in Qatar. Newly emerging class of anti-diabetic drugs focuses on SGLT inhibition were observed to reduce CVDs risks in diabetic patients. Up to date, the mechanism contributing to the $\mathrm{CV}$ benefits remains unrevealed. Zebrafish embryos were treated with Aristolochic Acid to induce heart failure then treated with Empagliflozin to determine its beneficial effect. Furthermore the expression of SGLT1 \& 2 were determined in the hearts of zebrafish. SGLT2 was expressed more then SGLT1 in the heart and whole embryo. Empa significantly improved the zebrafish embryo's cardiac health after induction of heart failure.

\section{Introduction}

Empagliflozin was shown nto improve the cardiovascular (CV) complication. Yet, the exact mechanism of Empagliflozin on $\mathrm{CV}$ tissues remains unclear. Therefore, this research aims to:

- Determine the expression level of SGLT1 and SGLT2 in zebrafish.

- Determine the toxicity of Empa on zebrafish.

- Analyze the impact of Empagliflozin in improving the cardiac function in zebrafish.

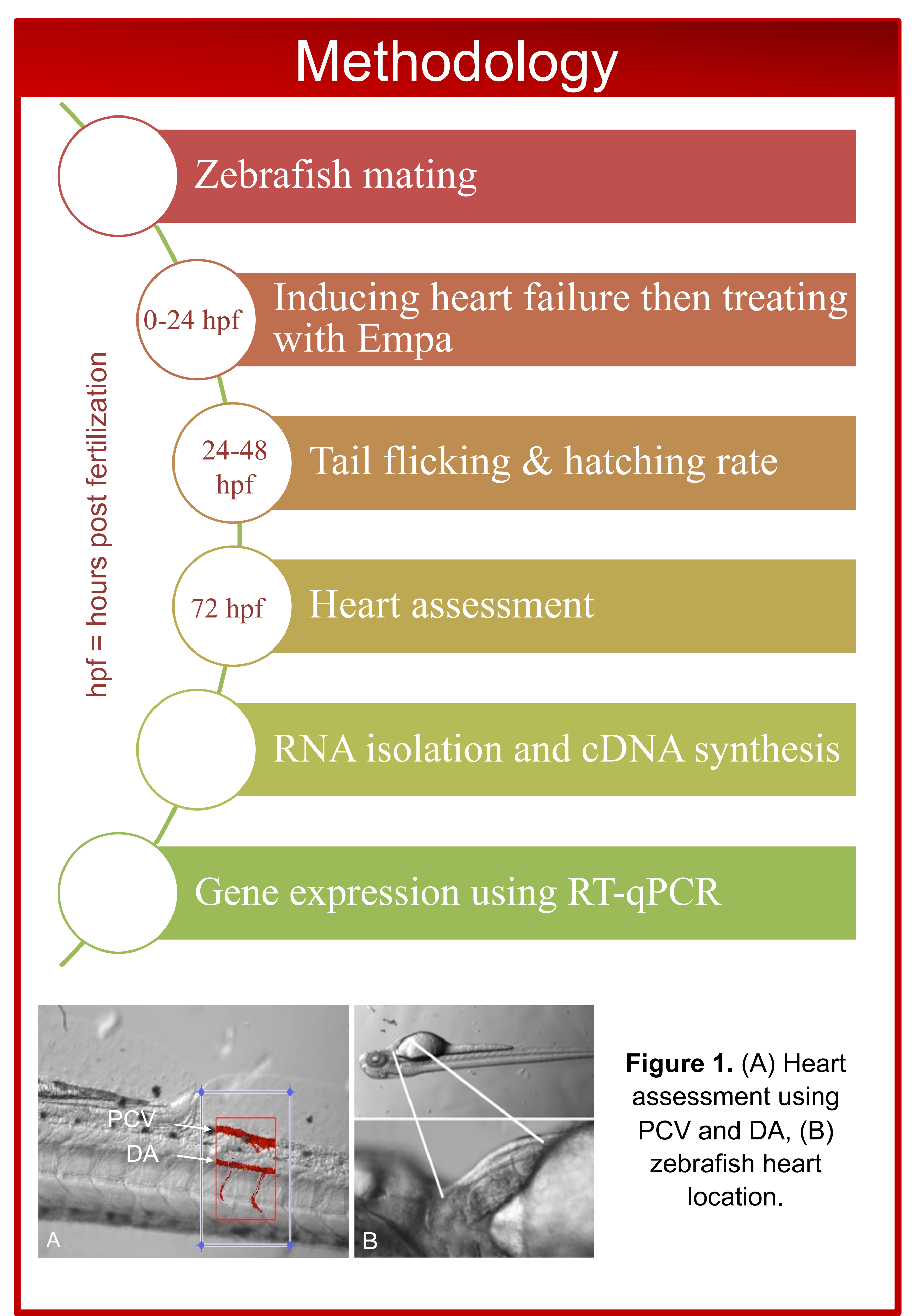

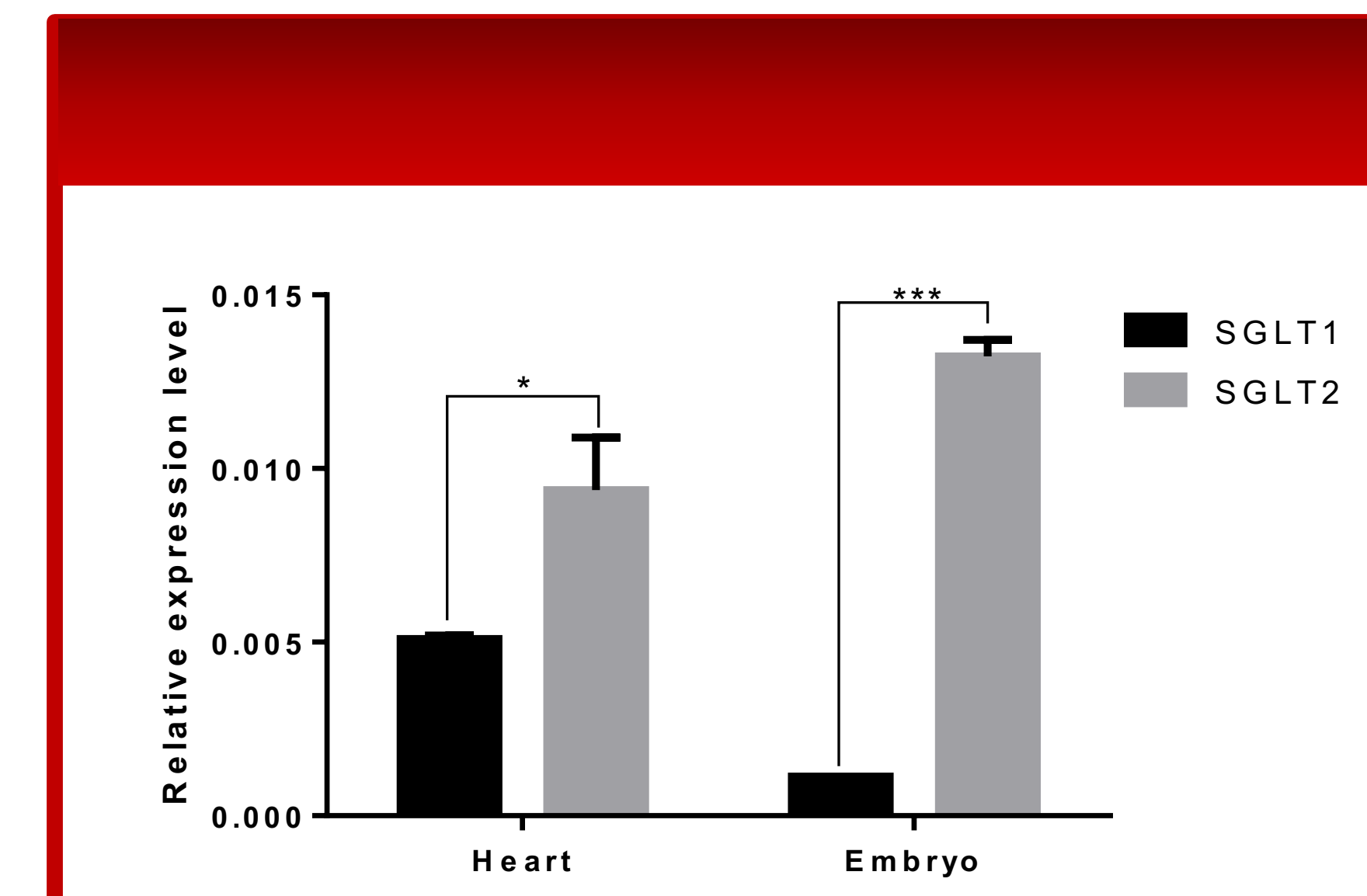

Results

Figure 1: SGLT-1 and SGLT-2 Expression in Zebrafish Using qPCR. RNA was isolated from hearts of zebrafish embryos and whole embryos then reverse transcribed to cDNA and subjected to QPCR analysis. Analysis was by one-way-ANOVA with sidak post hoc test to compare groups. Data is presented as mean \pm SEM. $N=3$ per group

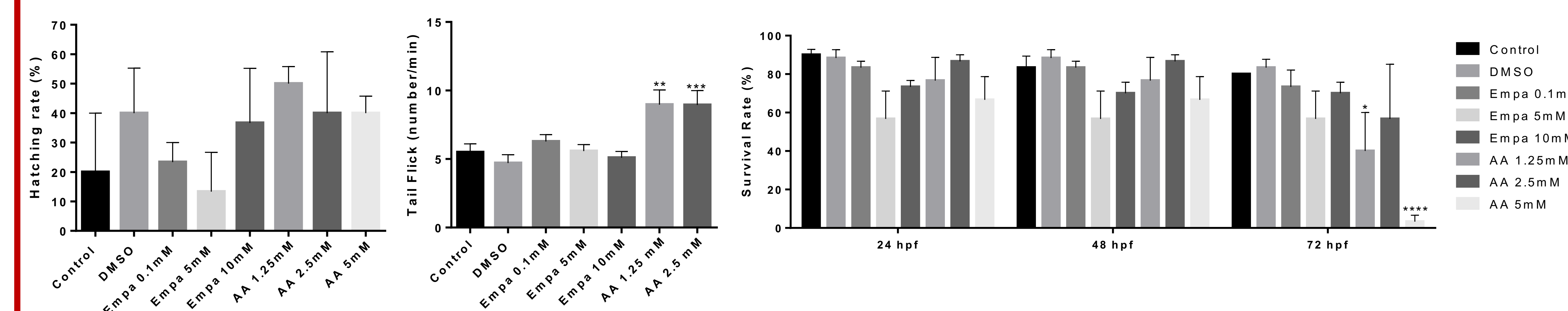

Figure 2: Hatching Rate measured Figure 3: Tail flicking activity of Figure 4: Comparison in Survival Rate as Percentage in Toxicity at 48 hpf for Toxicity Study. embryos at 24 hpf for Toxicity Study Study

Embryos were treated at 0 hour post Embryos were treated at 0 hour post Embryos were treated at 0 hour post fertilization (hpf) with PTU fertilization (hpf) with PTU water as fertilization (hpf) with PTU water as control, vehicle control containing control, vehicle control containing water as control, vehicle control
$0.1 \%$ DMSO (DMSO), Empa and 0.1\% DMSO (DMSO), Empagliflozin and Empa and AA concentrations. AA

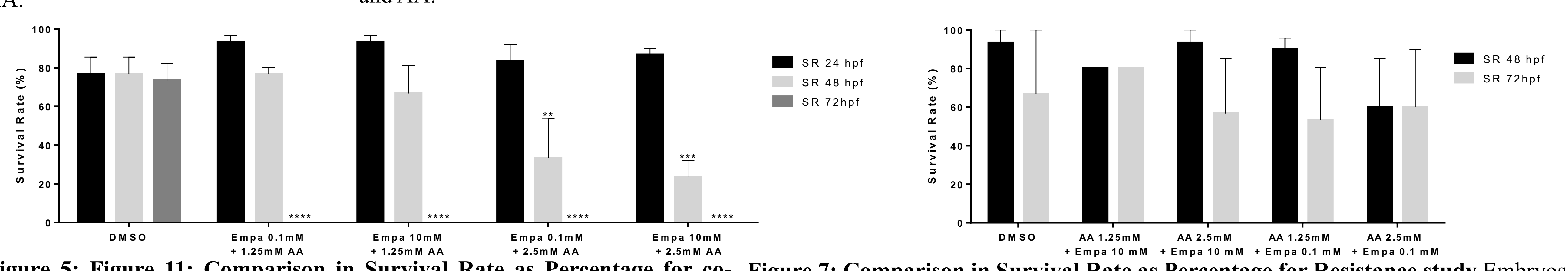

Figure 5: Figure 11: Comparison in Survival Rate as Pen treatment study. Embryos were treated at 0 hour post fertilization (hpf) with were treated at 0 hour post fertilization (hpf) with vehicle control containing $0.1 \%$ DMSO ( or $5 \mathrm{mM}$ for 8 hours followed by aristolochic acid (AA) at 1.25 or $2.5 \mathrm{mM}$ for Empagliflozin (Empa) 0.1 or $5 \mathrm{mM}$ after 24 hrs for 32 hours. analysis was done at 48 and 24 hours at 24,48 and $72 \mathrm{hrs}$. Treatment with $0.1 \mathrm{mM}$ Empa $+2.5 \mathrm{mM}$ AA 72 hrs post treatment. No significant difference was observed. caused a significant decrease in survival rate. All treatments cause a death in all
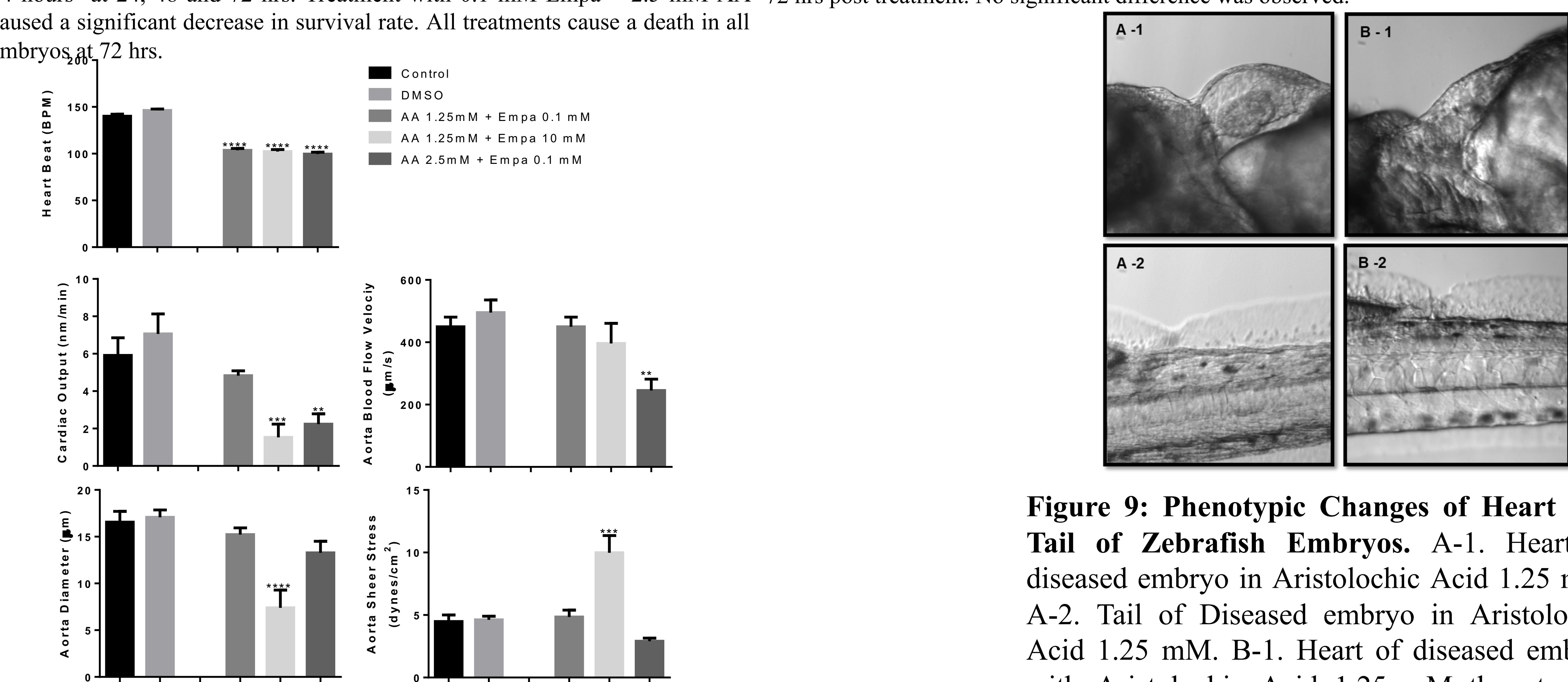

Figure 9: Phenotypic Changes of Heart and Tail of Zebrafish Embryos. A-1. Heart of diseased embryo in Aristolochic Acid $1.25 \mathrm{mM}$. A-2. Tail of Diseased embryo in Aristolochic Acid $1.25 \mathrm{mM}$. B-1. Heart of diseased embryo with Aristolochic Acid $1.25 \mathrm{mM}$ then treated Figure 8: Empagliflozin Effect on Cardiac Injury Markers. Embryos were with Empagliflozin 0.1mM. B-2. Tail of embryo treated at 0 hour post fertilization (hpf) with vehicle control containing $0.1 \%$ diseased with Aristolochic Acid $1.25 \mathrm{mM}$ then DMSO (DMSO), aristolochic acid (AA) at 1.25 or $2.5 \mathrm{mM}$ followed by treated with Empagliflozin $0.1 \mathrm{mM}$. Empagliflozin (Empa) 0.1 or $5 \mathrm{mM}$ after $24 \mathrm{hrs}$. Analysis was done at $72 \mathrm{hrs}$ post treatment. Heartbeat was significantly lower in all treated groups.

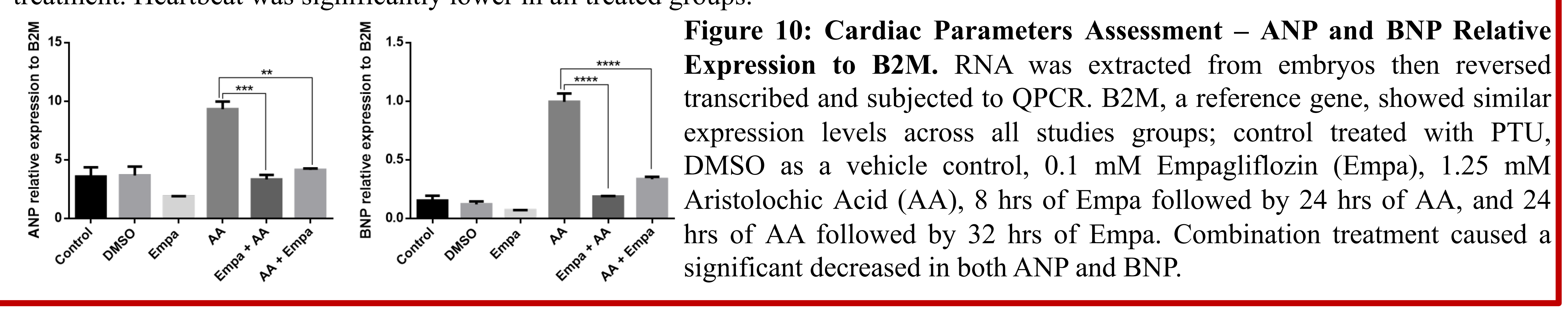

Conclusion

In conclusion, this study showed that Empagliflozin is effective in protecting or treating the cardiac function from cardiomyopathies in the zebrafish. This was evident by the improvement of the cardiac parameters after injury as well as the expression level of cardiac injury markers. The study showed that SGLT2 is highly expressed in the hearts which could mean that the effect of Empa in the cardiac tissue is direct through its target SGLT2. 